\title{
Die Rollen des Ganzen und der Teile in bundesstaatlichen Territorial- reformen: Ein Verfassungsvergleich zur Länderneugliederung*
}

\author{
Uwe Leonardy ${ }^{1}$
}

\section{Die Relevanz von territorialen Reformen für die Erneuerung von föderalen Systemen und besonders für die des deutschen}

Der Begriff der Schuldenbremse ist zwar mittlerweile zu einer europäischen Vokabel geworden. Er entstammt aber bekanntlich der deutschen so genannten Föderalismus-Reform II von $2009^{2}$, mit der insbesondere 3 festgelegt wurde, dass ab 2020 für die Länder grundsätzlich keine Einnahmen aus Krediten mehr zulässig sind. Diese Bestimmung wirft besonders für die finanzschwachen Länder schon heute ihre Schatten voraus, denn es ist fraglich, ob sie nach diesem Datum angesichts der beschriebenen Verfassungslage auch dann noch lebensfähig sein werden, wenn nicht der - unwahrscheinliche - Fall einer völligen Revision des Finanzausgleichs eintritt. Damit stellt sich mit zunehmendem Zeitablauf die Existenzfrage für diese Länder und insgesamt die Frage der Länderneugliederung neu und mit anwachsender Schärfe. Wie zu zeigen sein wird, ist eine solche Neugliederung allerdings mit dem dafür vorgesehenen verfassungsrechtlichen Instrumentarium des Art. 29 GG wegen des Fehlens einer Entscheidungsrolle des Bundes oder einer an ihre Stelle tretenden Regelung in Konflikten seiner Teile in praxi inoperabel. Es empfiehlt sich also immer stärker, dieses Instrumentarium in den Rollen des Ganzen und seiner Teile mit dem der wichtigsten anderen Bundesstaaten zu vergleichen, um festzustellen, wo und wie gewichtig seine Schwachstellen sind und mit welchem anderen Konzept sie behoben werden könnten.

Dabei ist im Verfassungsvergleich zunächst festzuhalten, dass die Erzielung föderaler Territorialreformen generell eine große Bedeutung für das Gelingen von Reformen und Erneuerungen bundesstaatlicher Systeme haben kann. Der Hauptgrund hierfür liegt darin, dass ein grundlegendes Erfordernis für das Funktionieren von Bundesstaaten in einem angemessenen Gleichgewicht in den Beziehungen auf der einen Seite zwischen dem Zentrum und der Gesamtheit der das System konstituierenden Teile (Regionen) wie auf der anderen

* Grundlage dieses Beitrags ist ein Referat des Verfassers mit dem Titel „The Roles of the Whole and the Parts in Federal Territorial Reforms", das auf dem XXI. Weltkongress der International Political Science Association (IPSA) 2009 in Santiago de Chile gehalten wurde; http://paperroom.ipsa. org/papers/view/698 (Abruf am 15. Februar 2013). Der Verfasser schuldet besonderen Dank Prof. Ronald L. Watts, Kingston (Kanada) für Anregungen nach Durchsicht des Referatsmanuskripts und Prof. Sonja Wältti, Washington D.C. (USA) für Kritik und Vorschläge in der Diskussion darüber während des IPSA-Kongresses.

1 Der Autor war mit der Thematik des Beitrags beruflich befasst als Verbindungsbeamter des Bundeskanzleramts zur Sachverständigenkommission für die Neugliederung des Bundesgebiets beim Bundesminister des Innern (der so genannten Ernst-Kommission) von 1970 bis 1973 . Als Referatsleiter in der Vertretung Niedersachsens beim Bund war er 1975/76 an der Novellierung des Art. 29 GG und von 1991 bis 1993 an den Arbeiten der Verfassungskommission des Bundesrates sowie der Gemeinsamen Verfassungskommission von Bundestag und Bundesrat beteiligt. Zu seinen Publikationen im Themenfeld; vgl. http://www.uwe-leonardy.de.

2 57. Gesetz zur Änderung des GG vom 31. Juli 2009; BGBl. I, S. 2247.

3 Durch die Art. 143 d Abs. 1 Satz 4 in Vbdg. mit Art. 109 Abs. 3 Satz 5 GG. 
Seite auch zwischen den Regionen selbst besteht. Die häufigsten Gründe für die Reform oder für die Erneuerung föderaler Systeme haben ihre Wurzeln in Ungleichgewichten wirtschaftlicher, finanzieller und/oder administrativer Art zwischen dem Zentrum und der Gesamtheit der Regionen. In den meisten Fällen sind diese selbst wiederum verursacht durch Ungleichgewichte zwischen den Regionen in ihrem wirtschaftlichen, finanziellen und den daraus hervorgehenden Verwaltungs- und/oder Verfassungskapazitäten.

Solche regionalen Imbalancen stehen in einem engen Zusammenhang mit zu großen Unterschiedlichkeiten in den Größen der Regionen und nicht selten auch mit zu hohen Zahlen der Regionen. Dementsprechend ist eine angemessene Territorialstruktur, die solche Ungleichgewichte verhindern kann, ein wichtiger Faktor für die Vermeidung von Schwächen des föderalen Systems in dem jeweiligen Land als solchem und ebenso für die Vermeidung einer Überlastung des Systems durch zu zahlreiche und zu schwerwiegende Konflikte. Deshalb wird die Erzielung einer solchen Territorialstruktur oft ein wesentliches Ziel von Bemühungen für die Erneuerung des bundesstaatlichen Systems sein müssen. Ein sogar noch größeres Gewicht dieser Notwendigkeit ist in Staaten zu beobachten, in denen die Gleichwertigkeit der Lebensverhältnisse zwischen den Regionen eine Verfassungsforderung ist wie zum Beispiel in Deutschland. Dementsprechend führt jene Notwendigkeit unvermeidbar eher zur Erhöhung als zur Verminderung von Ungleichgewichten und eher zur Verstärkung als zur Verminderung von Intransparenz, wenn durch ihre Vernachlässigung Versuche zu föderaler Reform beschädigt werden, wie es in einer Fallstudie über Deutschland in einem späteren Stadium dieses Beitrages zu zeigen sein wird.

Über die faktischen Hintergründe der auseinander klaffenden Zahlen von konstituierenden Einheiten und über die Wirkungen der Unterschiede in ihrer Größe sowie Wirtschaftsund Finanzkraft hat Ronald L. Watts überaus gründliche Forschungsarbeiten betrieben und über alle dazu relevanten Fakten in seinem Buch „Comparing Federal Systems“ berichtet, das inzwischen zu einem der Klassiker in der Vergleichenden Föderalismuslehre geworden ist. ${ }^{4}$ Daher brauchen hier keine Einzelheiten dargelegt zu werden, so dass ein kurzer Überblick über die Bereiche von Größe sowie von Wirtschafts- und Finanzkraft ausreichend sein wird.

\section{Untersuchte Bundesstaaten}

Bevor dies aber unternommen werden kann, wird es notwendig sein, die Bundesstaaten zu benennen, die hier untersucht werden sollen. Für ihre Auswahl sind Watts' Kategorien der „ausgereiften Föderationen“, der „sich entwickelnden Föderationen“, der „Mikroföderationen“, der „konföderal-föderalen Mischformen“ und der „postkonflikt-föderalen Experimente" verwendet worden. Die letztgenannten drei Gruppen wurden ausgeschlossen ${ }^{5}$, so dass sich die Untersuchung auf die „ausgereiften“ und die „sich entwickelnden“ Bundesstaaten konzentriert. Dies sind - aufgeführt in der Reihenfolge des Alters ihrer Verfassungen:

Vgl. Ronald L. Watts, Comparing Federal Systems, Montréal / Kingston 2008, S. 29 - 61, S. 72 ff. Mithin als „Mikroföderationen“ die Föderierten Staaten von Mikronesien, die Republik von Belau, die Föderation von St. Kitts und Nevis und die Union der Comoren, als „konföderal-föderale Mischformen“ die Vereinigten Arabischen Emirate und die Europäische Union sowie als „postkonflikt-föderale Experimente" die Republik Bosnien und Herzegowina, die Republik Sudan, die Republik Irak und die Demokratische Republik Kongo. Vgl. ebenda, S. 52 - 61. 
1. die Vereinigten Staaten von Amerika (1787),

2. die Schweizerische Konföderation (1848, revidiert 1999),

3. Kanada (1867),

4. das Commonwealth von Australien (1901),

5. die Vereinigten Mexikanischen Staaten (1917),

6. die Bundesrepublik Österreich (1920, revidiert 2008),

7. die Bundesrepublik Deutschland (1949),

8. die Republik Indien (1950),

9. die Föderation von Malaysia (1963),

10. die Islamische Republik Pakistan (1973),

11. das Königreich Spanien (1978),

12. die Föderative Republik Brasilien (1988),

13. das Königreich Belgien (1994),

14. die Russische Föderation (1994),

15. die Argentinische Republik (1994),

16. die Föderale Demokratische Republik von Äthiopien (1994),

17. die Republik Südafrika (1996),

18. die Föderale Republik Nigeria (1999) und

19. die Bolivarische Republik Venezuela (1999).

\section{Rangfolge territorialer Ungleichgewichte}

Für die Zwecke dieser Untersuchung dürften die relevantesten statistischen Zahlen diejenigen sein, aus denen die Unterschiede zwischen den größten und den kleinsten konstituierenden Einheiten in Prozentsätzen der Gesamtbevölkerung in dem jeweiligen Bundesstaat hervorgehen. Diese Unterschiede sind besonders wichtig, weil sie sehr stark die Grade der territorialen Ungleichgewichte in den untersuchten Bundesstaaten bezeichnen (vgl. Tabelle 1).

Diese Zahlen und ihre Rangfolge legen die folgenden Beobachtungen nahe: Es gibt in allen Bundesstaaten mehr oder weniger starke Unterschiedlichkeiten und dementsprechend Ungleichgewichte zwischen den konstituierenden Einheiten ausgedrückt in ihren Bevölkerungszahlen. Diese Ungleichgewichte können grob in drei Kategorien aufgeteilt werden:

(1.) Die erste Kategorie in den Rängen von 1 bis 6 (Pakistan bis Australien) ist durch hohe Disparitäten gekennzeichnet, weil mehr als ein Drittel der Gesamtbevölkerung - sowie in Pakistan und Belgien sogar mehr als die Hälfte davon - in der größten Einheit lebt.

(2.) Die zweite Kategorie (angeführt durch Deutschland und bis Österreich reichend) in den Rängen 7 bis 11 (mit Malaysia und Spanien beide im Rang 10) umfasst Bundesstaaten, in denen die größten Einheiten etwa ein Fünftel der Gesamtbevölkerung ausmachen, während die kleinsten - mit zwei Ausnahmen - weniger als ein Prozent von ihr haben. Die Unterschiede, die ihre Rangfolge kennzeichnen, liegen alle zwischen etwa 20 und 17 Prozent.

(3.) Die dritte und letzte Kategorie in den Rängen 12 bis 17 (Indien bis Nigeria) enthält alle bevölkerungsreichsten Föderationen (mit Ausnahme von Pakistan), aber zugleich gehören zu ihr auch alle Bundesstaaten mit den größten Bevölkerungszahlen in den konstituierenden Einheiten. Diese Doppeleigenschaft ist der Grund für die Tatsache, dass die Unter- 


\begin{tabular}{|c|c|c|c|c|c|c|}
\hline Rang & Bundesstaat & $\begin{array}{l}\text { Zahl der } \\
\text { Einheiten }\end{array}$ & $\begin{array}{c}\text { Gesamt- } \\
\text { bevölkerung } \\
\text { (in Millionen) }\end{array}$ & $\begin{array}{l}\text { Größte } \\
\text { Einheit* }\end{array}$ & $\begin{array}{l}\text { Kleinste } \\
\text { Einheit* }\end{array}$ & $\begin{array}{l}\text { Unter- } \\
\text { schiede* }\end{array}$ \\
\hline 1 & Pakistan & 4 & 144,9 & 55,6 & $-0,6$ & $=55,0$ \\
\hline 2 & Belgien** & 3 & 10,6 & 57,8 & $-9,7$ & $=48,1$ \\
\hline 3 & Argentinien & 23 & 36,3 & 38,1 & $-0,5$ & $=37,6$ \\
\hline 4 & Kanada & 10 & 30,0 & 38,0 & $-0,5$ & $=37,5$ \\
\hline 5 & Äthiopien & 9 & 67,3 & 35,2 & $-0,3$ & $=34,9$ \\
\hline 6 & Australien & 6 & 18,8 & 33,6 & $-2,4$ & $=31,2$ \\
\hline 7 & Deutschland & 16 & 82,4 & 21,6 & $-0,8$ & $=20,8$ \\
\hline 8 & Südafrika & 9 & 45,6 & 21,0 & $-1,8$ & $=19,2$ \\
\hline 9 & Schweiz & 26 & 7,4 & 17,0 & $-0,2$ & $=16,2$ \\
\hline 10 & Malaysia & 13 & 23,3 & 18,0 & $-0,9$ & $=17,1$ \\
\hline 10 & Spanien & 17 & 44,1 & 17,8 & $-0,7$ & $=17,1$ \\
\hline 11 & Österreich & 9 & 8,3 & 20,0 & $-3,3$ & $=16,7$ \\
\hline 12 & Indien & 28 & $1.028,6$ & 16,2 & $-0,5$ & $=15,7$ \\
\hline 13 & Mexiko & 31 & 103,3 & 13,6 & $-0,5$ & $=13,1$ \\
\hline 14 & Venezuela & 23 & 22,8 & 12,9 & $-0,3$ & $=12,6$ \\
\hline 15 & USA & 50 & 281,4 & 12,0 & $-0,1$ & $=11,9$ \\
\hline 16 & Russland & 86 & 144,0 & 7,1 & $-0,01$ & $=7,1$ \\
\hline 17 & Nigeria & 36 & 132,8 & 6,5 & $-1,1$ & $=5,4$ \\
\hline \multicolumn{7}{|c|}{ 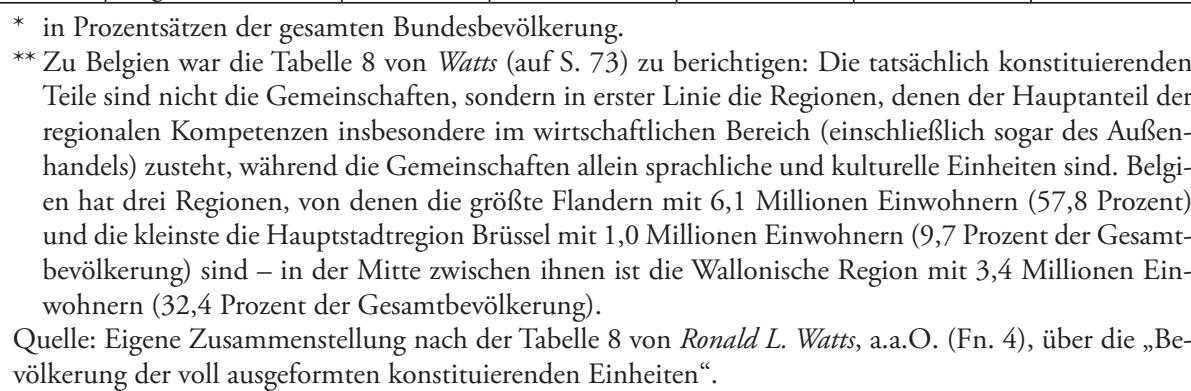 } \\
\hline
\end{tabular}

schiede, die ihre Rangfolgen ausmachen (von etwa 15 bis etwa fünf Prozent), verhältnismäßig klein sind im Vergleich zu den anderen zwei Kategorien und trotz der hohen Gesamtbevölkerungszahlen in den hier betroffenen Bundesstaaten.

In einem übergreifenden Vergleich fällt des Weiteren auf, dass Deutschland die zweite Gruppe anführt und dass diese Föderation zugleich (mit Ausnahme von Belgien als einem strukturellen Sonderfall) der europäische Bundesstaat mit dem höchsten Rang von territorialen Ungleichgewichten ist. Zu dieser Tatsache wird in einem späteren Stadium zurückzukommen sein.

\section{Auswirkungen der Ungleichgewichte}

Alle diese Ungleichgewichte haben natürlich ihre oft sogar schwerwiegenden Auswirkungen auf das Funktionieren der jeweiligen Bundesstaaten. Diese können von wirtschaftlicher und 
aus ihr sich ergebender finanzieller wie sozialer Art sein, wenn sie nicht - wie in einigen Fällen - in einem bestimmten Ausmaß durch finanzielle Ausgleichssysteme gemildert werden, die ihrerseits jedoch in starkem Ausmaß die Motivationen für Wettbewerb zwischen den Einheiten vermindern. Die für die politische, administrative und wirtschaftliche Funktionsweise jedes Bundesstaates relevante Tatsache ist, „dass innerhalb einer Föderation große Unterschiede zwischen den konstituierenden Einheiten im Hinblick auf ihre Kapazität zur Aufgabenerfüllung und in ihrem Einfluss auf die föderale Politikgestaltung herrschen können"6.

Obwohl es keinen direkten Bezug zwischen den Bevölkerungszahlen und der Wirtschaftsund Finanzkraft konstituierender Einheiten gibt, ist dennoch die Aussage notwendig, dass beide Faktoren eng miteinander verbunden sein können, wenn und wo auch immer Synergien aus der Zusammenlegung von kleinen und/oder mittelgroßen Einheiten zur Debatte stehen. Darüber hinaus ist das „Argument“, dass zwei Arme zusammen noch keinen reichen Mann ausmachen, in diesem Zusammenhang sehr oberflächlich, weil es solche Synergien vernachlässigt.

Aufgrund all dieser Tatsachen und Prozesse ist ein statistischer Beweis jenseits von Bevölkerungszahlen sowohl schwierig als auch problematisch zu führen. Zuerst einmal sind solche wirtschaftlichen und sozialen Statistiken sehr häufig in erheblichem Maß schnellen Wechseln unterworfen, und zweitens können sie sicher nicht in angemessener Tiefe innerhalb des Rahmens und der Grenzen dieser Untersuchung diskutiert werden. Darüber hinaus dürfte es als eine Binsenweisheit jenseits der Notwendigkeit von statistischen Beweisen erscheinen, dass die angeführten Ungleichgewichte zwischen den Einheiten von Föderationen in der Tat existieren und dass dementsprechend „Variationen innerhalb jedes Bundesstaats in Begriffen ihrer relativen Wirtschafts- und Finanzkraft seit jeher ein Faktor waren [und sind], der den Einfluss bestimmter konstituierender Einheiten in der bundespolitischen Dynamik bestimmt"7.

\section{Die Rollen des Ganzen und der Teile in den verfassungsrechtlichen Reformverfahren von Föderationen}

Dementsprechend trifft es sowohl im Hinblick auf die Potenziale des Funktionierens eines bundesstaatlichen Systems als auch im Hinblick auf die Geltungskraft seiner Einheiten zu, dass es in bundesstaatlichen Territorialreformen legitime Interessen sowohl des Ganzen wie der Teile an ausreichendem Einfluss auf und in solchen Verfahren gibt.

Es ist daher zu untersuchen, wie und in welchem Ausmaß die relevanten Bestimmungen föderaler Verfassungen diese Interessen anerkennen und ihnen Rechnung tragen und ob solche Interessen in dem verfassungsrechtlichen Rahmen angemessen ausbalanciert sind.

$\mathrm{Zu}$ diesem Zweck sind die Bestimmungen in den Verfassungen für neue Umgrenzungen von Einheiten, über die Verschmelzung von bestehenden und über die Hinzufügung von neuen Einheiten zu dem jeweiligen Bundesstaat erfasst worden (während solche allein über Grenzberichtigungen nicht einbezogen worden sind). Diese Auszüge sind in der folgenden Tabelle in vier Gruppen auf der Basis ihrer Wesensmerkmale aufgelistet worden: 


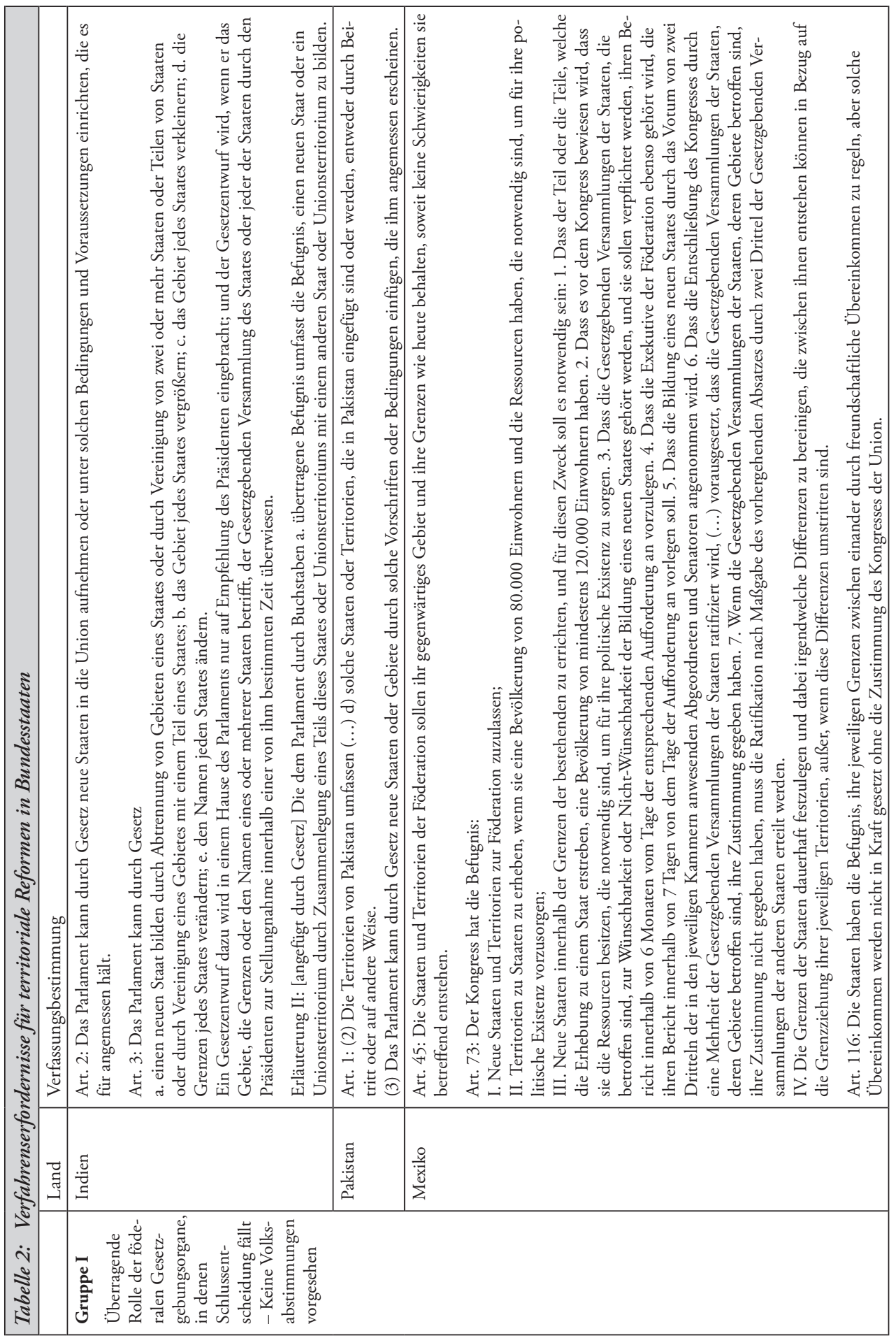




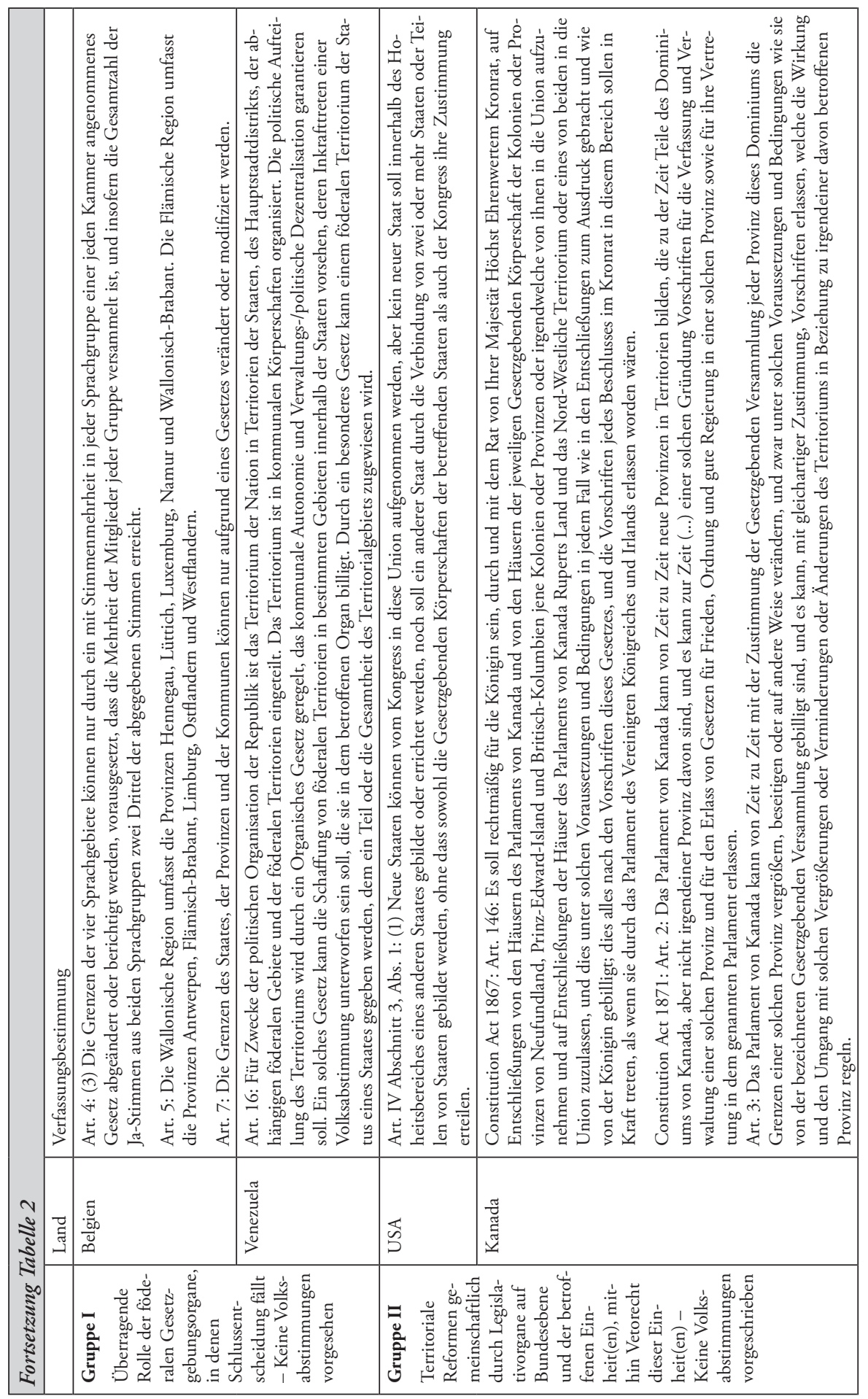




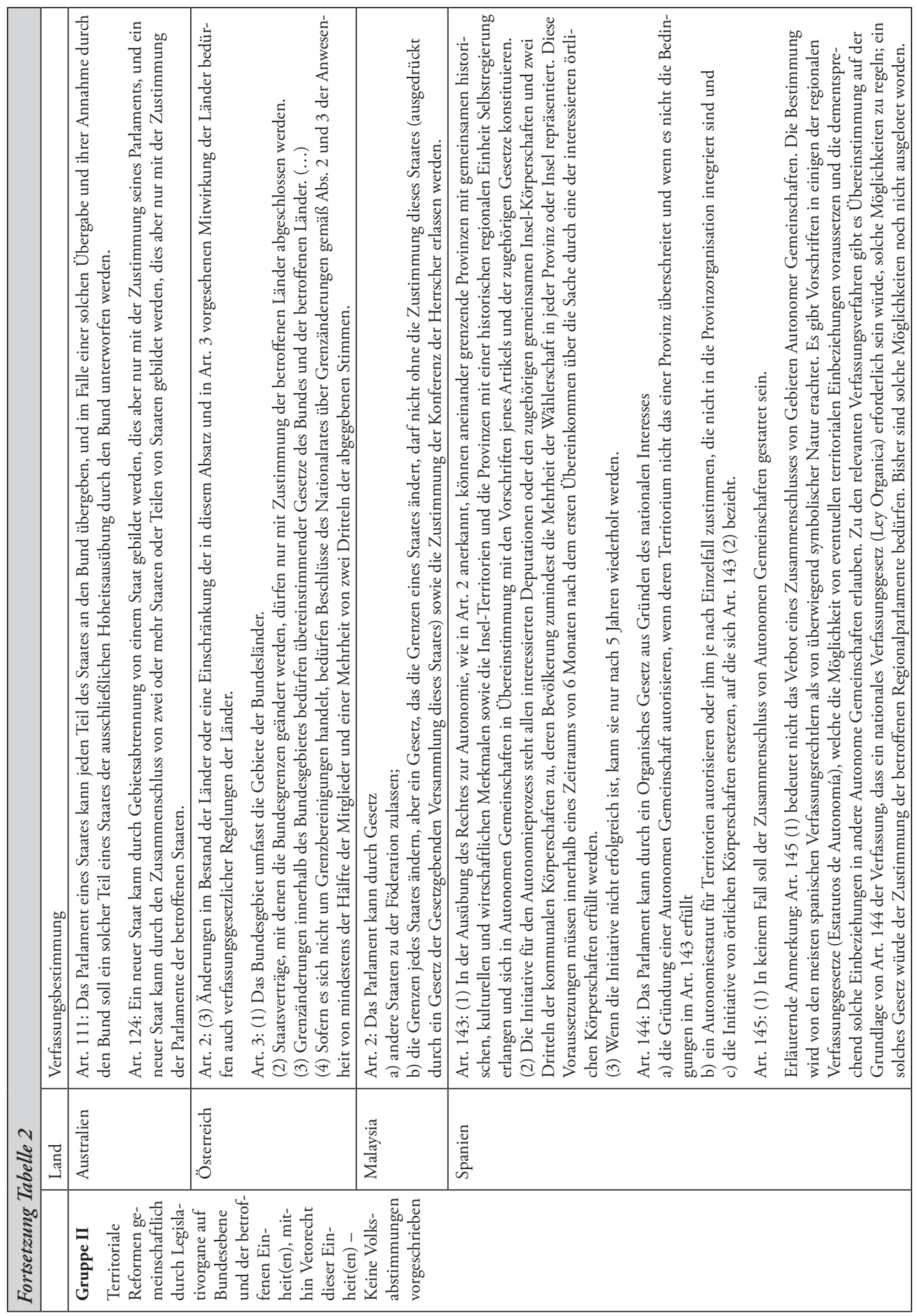




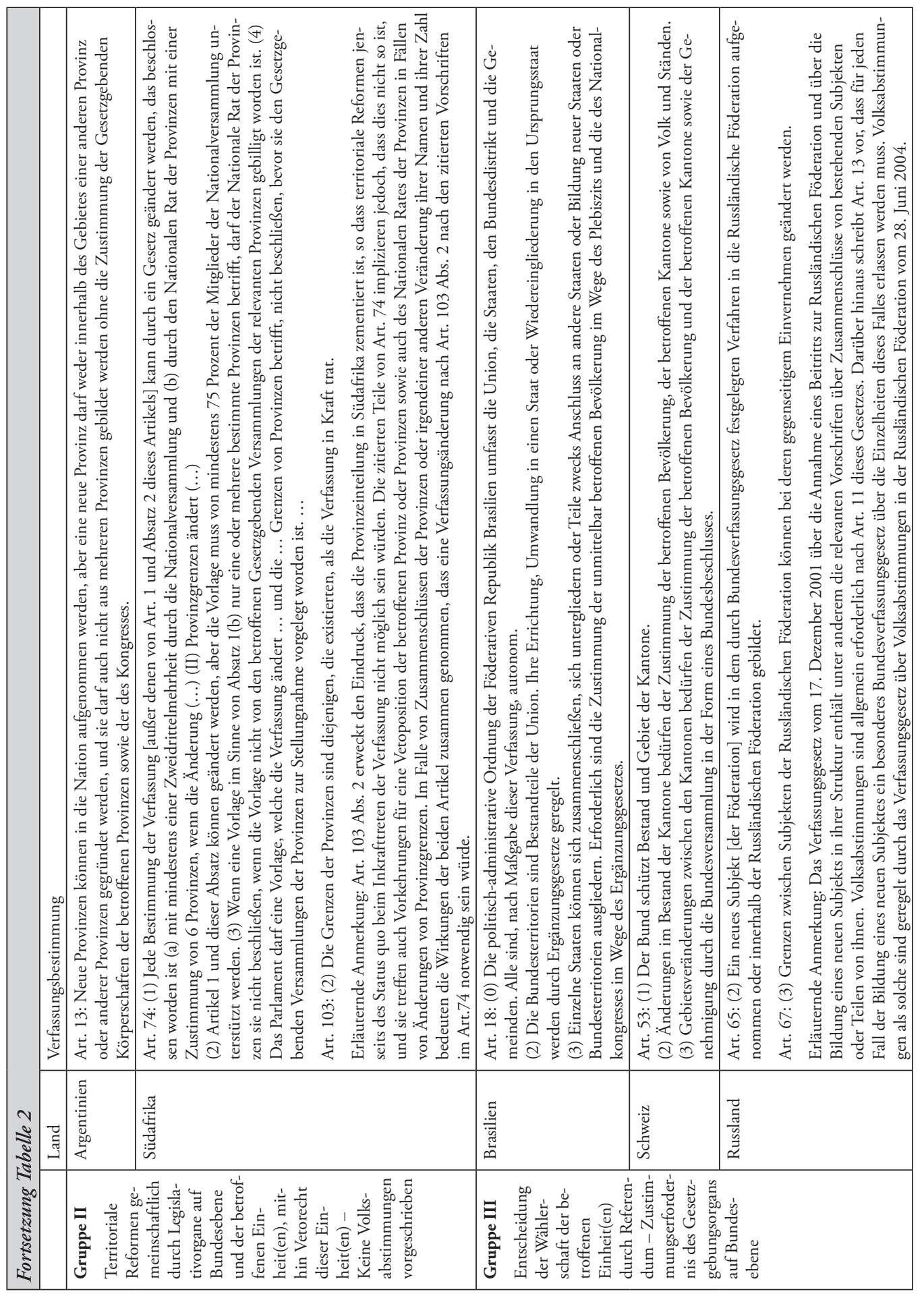




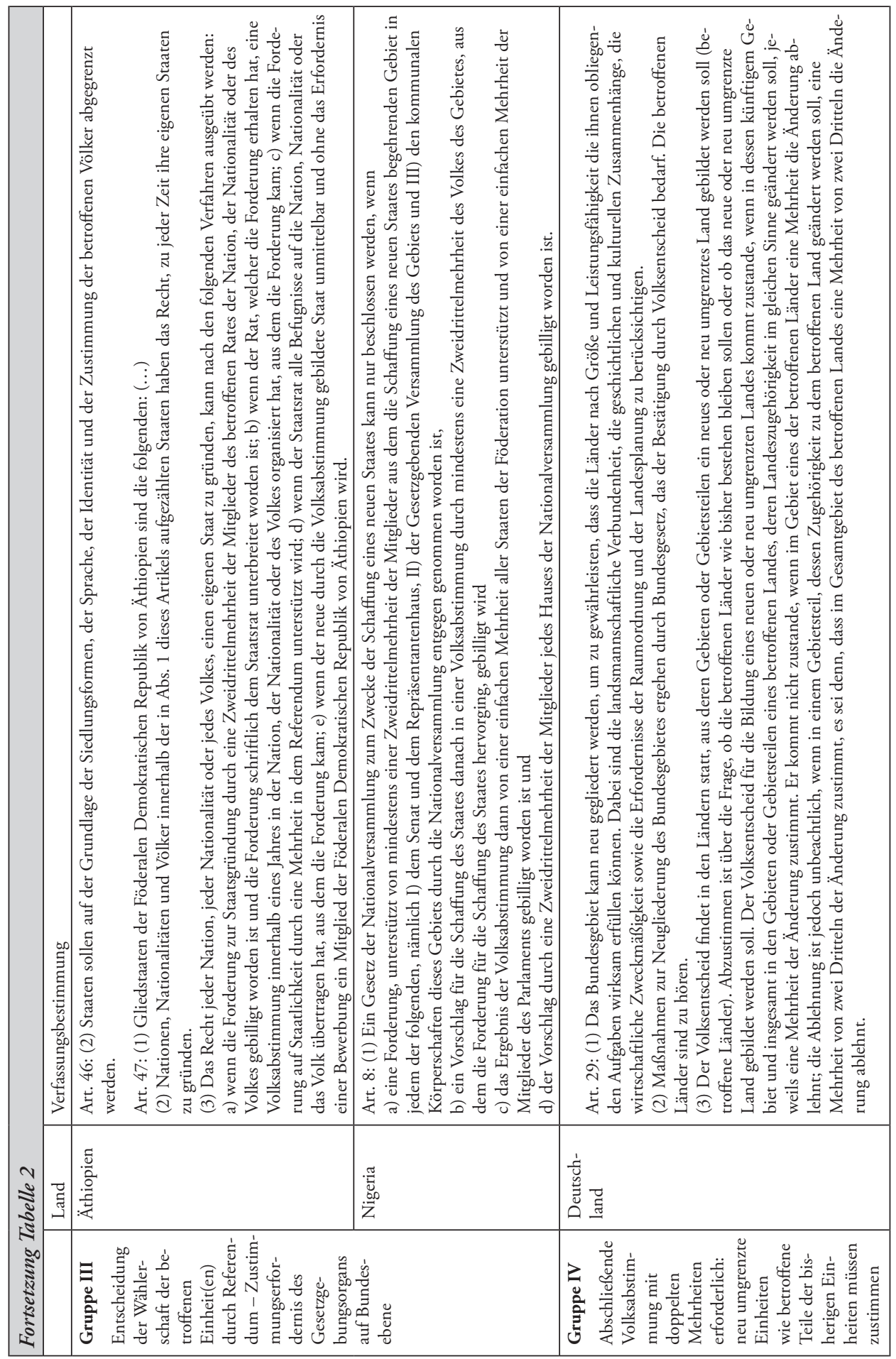




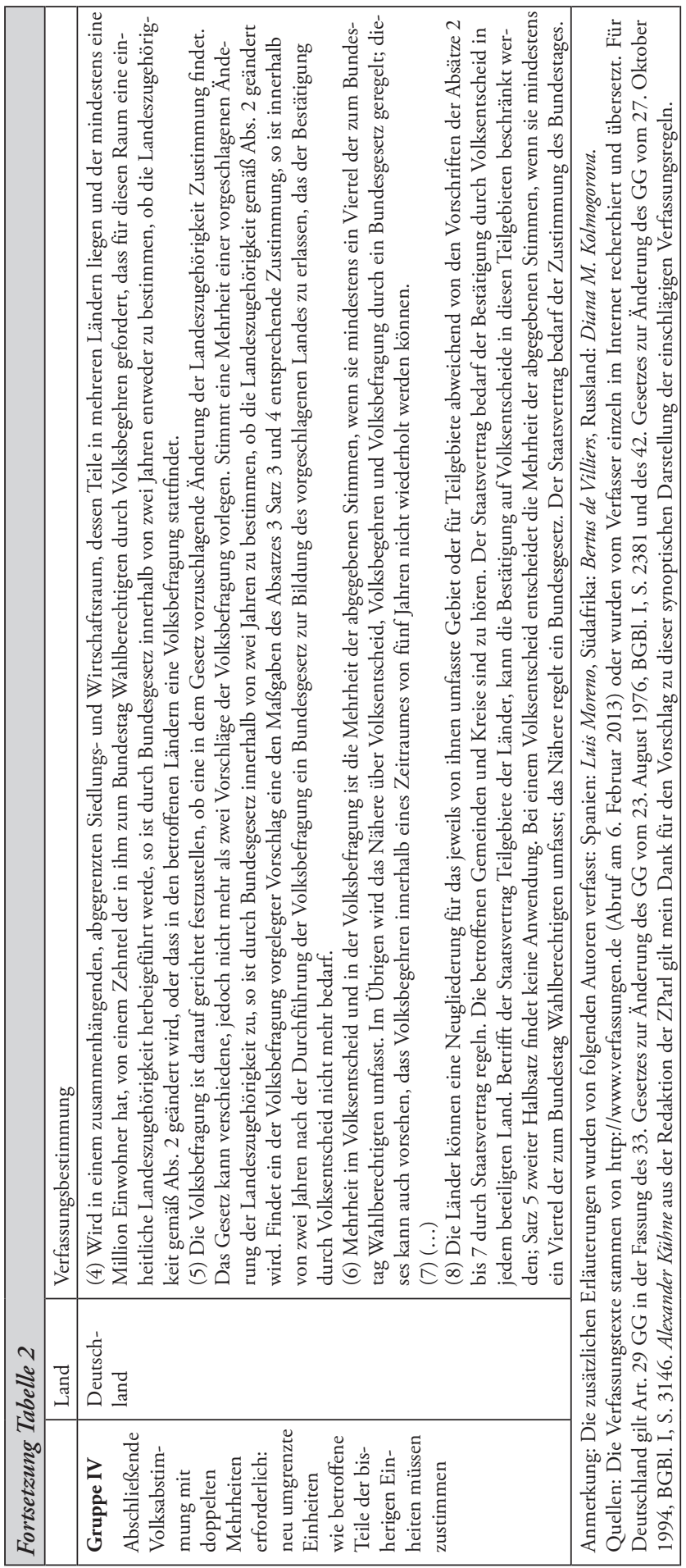




\section{Wesensmerkmale der exzerpierten relevanten Verfassungsvorschriften}

In einem Überblick mit einigen der wichtigsten Einzelheiten stellt sich das Bild bezogen auf die in der Tabelle 2 bezeichneten Gruppen wie folgt dar:

Zur ersten Gruppe gehören Indien, Pakistan, Mexiko, Belgien und Venezuela. Das indische Parlament hat volle Befugnisse zu territorialer Reform, wie es sie für richtig erachtet, während die Gesetzgebende Versammlung des betroffenen Staates nur „zur Stellungnahme“ innerhalb einer vom Präsidenten bestimmten Zeit gehört wird, und es sind in der Tat weitreichende neue Abgrenzungen auf dieser Grundlage bewirkt worden. ${ }^{8}$

Die pakistanischen Regeln (in Art. 1) ähneln den indischen, sind aber weit weniger detailliert.

In Mexiko kann der Kongress auch selbst territoriale Reformen veranlassen, aber dies nur unter bestimmten festgelegten Bedingungen. Die entsprechende Entschließung des Kongresses erfordert eine Ratifikation durch eine Mehrheit der Gesetzgebenden Körperschaften der Staaten einschließlich der betroffenen, aber ihr Nichteinverständnis kann durch zwei Drittel der Gesetzgebenden Körperschaften der anderen Staaten beseitigt werden.

In Belgien gibt es spezifische Regeln nur für die Veränderung von Grenzen der sprachlich determinierten Gemeinschaften mit dem Erfordernis besonderer Mehrheiten in den Sprachgruppen im Parlament, während die Abgrenzung der Provinzen (und damit auch der Regionen) nur durch Gesetz geändert oder modifiziert werden kann.

In Venezuela ist die politische Einteilung des Territoriums durch ein Organisches Gesetz mit bestimmten Garantien zu regeln, und eine Volksabstimmung ist nur erforderlich für die Schaffung von Bundesterritorien innerhalb der Staaten.

Die zweite Gruppe wird gebildet durch die Vereinigten Staaten, Kanada, Australien, Österreich, Malaysia, Spanien, Argentinien und Südafrika.

In allen diesen Staaten bedarf es zu territorialen Reformen übereinstimmender Beschlüsse der Legislativorgane des Bundes und der betroffenen Einheiten, denen aber Volksabstimmungen weder auf der Bundes- noch auf der Regionalebene zu folgen brauchen.

$\mathrm{Zu}$ Österreich ist jedoch bemerkenswert, dass territoriale Reformen ,auch verfassungsgesetzlicher Regelungen der Länder bedürfen“.

In Spanien und Südafrika sind die erforderlichen Regelungen durch Änderungen des nationalen Verfassungsrechts zu treffen, die aber ebenfalls nicht Volksabstimmungen zu unterwerfen sind; siehe zu beiden Staaten die erläuternden Anmerkungen.

In der dritten Gruppe sind die Schweiz, Brasilien, Russland, Äthiopien und Nigeria.

In der Schweiz werden sehr weitreichende Schritte für territoriale Veränderungen verlangt, aber die Rollen des Ganzen und der Teile sind (anders als in Deutschland, siehe unten) gleich stark in ihnen: Für die Schaffung neuer Kantone ist die Zustimmung der betroffenen Bevölkerung auch in Teilen der jeweiligen konstituierenden Einheit erforderlich (wie in Deutschland), aber zusätzlich ist neben der Zustimmung der Wählerschaft und der betroffenen Kantone auch die der Gesamtbevölkerung und aller Kantone erforderlich. Darüber hinaus muss die Verfassung selbst geändert werden, weil die Kantone in ihr aufgezählt sind. (Alles in allem waren dies die Gründe dafür, dass das Verfahren für die Schaffung des neuen Kantons Jura mit zahlreichen Volksabstimmungen von 1970 bis 1978 dauerte.) Für territo-

8 Vgl. Rekha Saxena, Situating Federalism: Mechanisms of Intergovernmental Relations in India and Canada, New Delhi 2006, S. 110 - 114. 
riale Veränderungen zwischen bestehenden Kantonen ist wiederum die Zustimmung der betroffenen Bevölkerungen und der betroffenen Kantone notwendig, und darüber hinaus muss das Bundesparlament ihnen zustimmen.

Annahme eines entsprechenden Bundesgesetzes durch Volksabstimmung in den betroffenen Staaten ist erforderlich in Brasilien und in Äthiopien.

In Russland verlangt die Bildung jedes neuen Subjekts der Föderation ein besonderes Bundesverfassungsgesetz, das eine Volksabstimmung vorsehen muss; zu Einzelheiten siehe die erläuternde Anmerkung hierzu.

In Nigeria ist eine Zweidrittelmehrheit in einer Volksabstimmung in dem betroffenen Gebiet erforderlich, der eine (einfache) Mehrheit der Staaten und wiederum eine Zweidrittelmehrheit in beiden Häusern der Nationalversammlung zustimmen muss, nachdem das Ergebnis der Volksabstimmung durch das Parlament des betroffenen Gebiets gebilligt worden ist.

In der vierten Gruppe ist Deutschland das einzige Land, und seine Regeln über Territorialreformen sind in der Tat einzigartig in einem ganz besonderen Sinn: Ohne dem Ganzen eine gleichrangige und erst recht nicht eine übergeordnete Rolle gegenüber den Teilen zu geben, erfordern diese Regeln doppelte Mehrheiten in Volksabstimmungen in den betroffenen Ländern und sogar auch in den Teilgebieten von ihnen im Falle eines Bundesgesetzes über die Neugliederung der Länder. Mehrheiten in Volksabstimmungen in jedem der betroffenen Länder sind notwendig im Falle eines Staatsvertrags über eine Territorialreform zwischen zwei oder mehr Ländern, die der Zustimmung des Bundestages unterliegt Die Wirkungen dieser Bestimmungen werden in der Fallstudie am Ende dieses Beitrags näher zu untersuchen sein.

In einer Zusammenfassung der Verfahrenserfordernisse für territoriale Reformen in bundesstaatlichen Verfassungen sind mithin die Interessen des Gesamtstaates gegenüber den Teilen am stärksten in Indien geschützt wie in unterschiedlichen Graden auch in den anderen Ländern der Gruppe I, während in allen anderen Föderationen die betroffenen konstituierenden Einheiten Vetorechte gegenüber dem Gesamtstaat haben, die in den Ländern der Gruppen III und IV durch Volksabstimmungen ausgeübt werden. Dies bedeutet, dass in allen Gruppen von II bis IV die Zustimmung der betroffenen Bevölkerung erforderlich ist, aber die Rollen des Ganzen gesichert sind, wenn auch in sehr unterschiedlicher Weise. Es gibt nur eine Ausnahme dazu, nämlich Deutschland im Falle eines Bundesgesetzes über die Länderneugliederung; hier gibt es keine Rolle des Ganzen gegenüber divergierenden Volksabstimmungen überhaupt.

Darüber hinaus ist nur in Deutschland und in der Schweiz das Prinzip der regionalen und plebiszitären Zustimmung so weit ausgeprägt worden, dass es sogar Teile der Bevölkerung in den betroffenen konstituierenden Einheiten betrifft, während in allen anderen $\mathrm{Zu}$ stimmungsfällen die Entscheidung durch die Gesamtbevölkerung dieser Einheiten selbst zu treffen ist. Mehr noch als das sind in Deutschland (und nicht einmal in der Schweiz) doppelte Mehrheiten für ein Referendum über ein Bundesgesetz erforderlich: eine im künftigen Gebiet „eines neuen oder neu umgrenzten Landes“ und eine zweite „insgesamt in den Gebieten oder Gebietsteilen eines betroffenen Landes, deren Landeszugehörigkeit im gleichen Sinne geändert werden soll“" (Art. 29 Abs. 3 Satz 3 GG). Durch diese Bestimmung werden dementsprechend sogar nur indirekt betroffene Teile der Wählerschaft in einem Land Inhaber eines Vetorechts. Auf diesem doppelten Weg hat Deutschland das Prinzip der Zustimmung der betroffenen Gebiete und der Teile in ihnen in sein Extrem mit dem Ergebnis ausgeprägt, das in der Praxis eine Reform unerreichbar ist. 


\section{Die Philosophie des regionalen Zustimmungsprinzips}

Diese Tatsache gibt Anlass zu Überlegungen und sogar zu Zweifeln über die zu Grunde liegende Philosophie des regionalen Zustimmungsprinzips. Seine Wurzeln liegen in einem ursprünglich spezifischen und damit legitimen Zweck von Föderalismus: der Intention, ethnische, kulturelle und/oder sprachliche Einzigartigkeit oder Unterschiedlichkeit in ihrem territorialen Zuschnitt zu schützen. In der Geschichte von Föderationen ist das sicherlich ein wichtiger Faktor gewesen, aber dennoch sind Zweifel gerechtfertigt, ob dieser Faktor unter allen Umständen vorrangig sein sollte oder dies tatsächlich auch ist. Zu allererst ist dies nicht so in einem Lande, das mit Recht ein Modell von Unterschiedlichkeiten genannt werden kann: Wie diese Untersuchung gezeigt hat, gibt es kein solches Vetorecht von territorial organisierter Unterschiedlichkeit in Indien. Darüber hinaus hat die Verleihung von Territorialität an Unterschiedlichkeit nicht notwendigerweise und immer friedenstiftende Wirkung. Es gibt andere Wege, Unterschiedlichkeit zu schützen, und der herausragende unter ihnen ist der Schutz von Menschen- und in besonderer Weise Minderheitenrechten durch die Gerichte. In Südafrika war dies zum Beispiel der entscheidende Grund dafür, die neuen neun Provinzen 1994 nicht auf der Grundlage von ethnischen oder anderen kulturellen Grundlagen zu schaffen und zu umgrenzen.

Die relevanteste Beobachtung im Zusammenhang dieser Untersuchung führt zu der Tatsache, dass die Ausstattung der betroffenen Teile mit einem nicht modifizierbaren Vetorecht in Territorialreformen die Bedeutung missachtet, die solche Reformen für die Lebensfähigkeit der Föderation als solche haben können. Aus diesem Grunde ist es nicht nur legitim, sondern auch notwendig, der Gesamtbevölkerung eines Bundesstaates eine angemessene Rolle in Bemühungen dieser Art zu geben.

Diese Notwendigkeit unter dem Vorwand zu begraben, dass sie die „Souveränität“ der Teile des Bundesstaates verletze, stellt eine Fehlanwendung des Konzepts der regionalen Souveränität dar. Dieses Konzept bedeutet, dass eine politische Einheit volle Befugnisse über alle ihre Kompetenzen hat. Indem sie eine Einheit in einer Föderation wird, übergibt diese Einheit aber mehr oder weniger große Teile jener Kompetenzen an die Föderation, und indem sie das tut, macht sie das Konzept der Souveränität durch und für sich selbst per definitionem zu einem überholten. Dementsprechend führt die missverstandene Überbetonung jenes Konzepts nicht selten zu Blockaden oder zumindest schweren Defekten von manchmal höchst notwendigen Reformen des gesamten bundesstaatlichen Systems, wenn der Gesamtstaat oder seine Wählerschaft keinen hinreichenden Einfluss auf solche Reformen hat.

Eine Fallstudie über Deutschland und über die Gründe von vielfach gescheiterten oder in sich widersprüchlichen bundesstaatlichen Reformen in seiner Geschichte ${ }^{9}$ kann dies sehr deutlich belegen.

9 Zur Geschichte dieser Reformbemühungen siehe Uwe Leonardy, Föderalismusreform ohne Länderneugliederung?, in: Frank Decker (Hrsg.), Föderalismus an der Wegscheide?, Wiesbaden 2004, S. 75 - 97; Uwe Leonardy, Die Neugliederung des Bundesgebietes: Auftrag des Grundgesetzes, in: Karl Eckert / Helmut Jenkins (Hrsg.), Föderalismus in Deutschland, Berlin 2001, S. 10 - 35. 


\section{Fallstudie Deutschland und ibre Bedeutung für den Untersuchungsgegenstand: Eine verfassungsunwürdige Verfassungsnorm als Kernproblem}

Um mit dem überraschendsten Aspekt der Geschichte von Territorialreformen angesichts der gegenwärtigen und oben beschriebenen Regeln zu beginnen: Sowohl in der Ursprungsversion von 1949 als auch nach der ersten Revision des Art. 29 GG von 1969 verlangte das Grundgesetz bis 1976, dass in Fällen von einander widersprechenden regionalen Referenden ein Volksentscheid im gesamten Bundesgebiet das letzte Wort haben sollte. Das war in der Tat der stärkste Ausdruck der Rolle des Gesamtstaats in solch einem Verfahren, so dass eine Regelung dieser Art kaum als eine Verletzung der Rechte der betroffenen Einheiten gelten konnte. Dies trifft umso mehr zu, als schon 1956 das Bundesverfassungsgericht aus Art. 29 GG die Forderung nach einem Gesamtkonzept für das ganze Bundesgebiet abgeleitet hatte. ${ }^{10}$

Der Wechsel zu genau dem entgegengesetzten Verfahren in den hier dargelegten gegenwärtigen Verfassungsregeln kam mit der dritten Revision von Art. 29 im Jahre 1976. Die Gründe für diese Revision brauchen hier nicht diskutiert zu werden. ${ }^{11}$ Das Ergebnis jedoch war, dass die Gegner einer Territorialreform als solcher dadurch obsiegten, dass sie die Funktion der gesamten Bundesbevölkerung als Schiedsrichter abschafften und jeden Reformversuch in einem höchst komplizierten Schema von voraussehbar einander entgegengesetzten Referenden sogar in den Teilen der betroffenen Einheiten erstickten. Dieses Schema war vor allem deshalb politisch erfolgreich, weil es gewünscht und gestützt durch den damaligen Regierungschef war: Bundeskanzler Helmut Schmidt hatte entschieden, dass der Status Hamburgs als eines Stadtstaates, aus dem er kam, unter allen Umständen erhalten bleiben sollte und dass er unberührt durch die damalige Debatte wie auch durch alle künftigen Debatten über Territorialreformen bleiben sollte. Das Ergebnis ist eine paradoxe Verfassungsnorm für die Verhinderung einer territorialen Reform und deutlich eben nicht für ihre Erzielung. Weil das genau so gewollt war, stellt der Art. 29 GG in seiner dadurch geprägten heutigen Fassung eine verfassungsunwürdige Verfassungsnorm dar. Das gilt noch weit mehr deshalb, weil er angesichts der notwendigen Zweidrittelmehrheit auch im Bundesrat nur unter großen politischen Schwierigkeiten wieder abänderbar ist. Ein späterer Zusatz im Jahre 1994 verbesserte dann zumindest die Verfahrenssituation dadurch, dass er es benachbarten Ländern ermöglichte, Staatsverträge über Zusammenschlüsse mit Zustimmung des Bundestages zu schließen (Art. 29 Abs. 8 GG). Aber das milderte die Situation politisch nicht wesentlich, weil es voraussetzte, dass die Führungen der betroffenen Länder freiwillig ihre „Throne“ in Verhandlungen über solche Verträge aufzugeben hatten, es sei denn, sie würden unter schwerwiegenden Druck aus der Bevölkerung und/oder der finanziellen Verhältnisse geraten.

Diese Lage, in der es keinen wie auch immer geratenen Bundesschiedsrichter wie vor 1976 gab, musste der Ausgangspunkt sein, als die Überlegungen über das begannen, was als weitreichende Reform des föderalen Systems im Jahre 2003 beabsichtigt war. Aber anstatt die Länderneugliederung hochrangig in der Agenda der Föderalismusreform I aufzuführen, wurde das gesamte Thema dort völlig durch seine Opponenten in den schwachen Ländern tabuisiert. Dies bezog sich insbesondere auf die Verfahrensregeln zu einer Territorialreform, und angesichts der dargestellten Inhalte dieser Regeln hatten die Opponenten natürlich ein

10 Vgl. BVerfGE 5, S. 34.

$11 \mathrm{Zu}$ Einzelheiten siehe die in Fußnote 9 zitierten Publikationen. 
leichtes Spiel, das sie sogar so weit trieben, mit dem Verlassen des Verhandlungstisches zu drohen, falls irgendwelche Teile des Problems und insbesondere diejenigen des Verfahrensrechts auch nur berührt werden würden. ${ }^{12}$ Die Situation war ähnlich in der Föderalismuskommission II, die nach ihrer Gründung im Jahre 2006 hauptsächlich mit Finanzfragen befasst war, obwohl ihr Auftrag durchaus eine Diskussion über die Verbesserung der Bedingungen für Zusammenlegungen von zwei oder mehr Ländern erlaubt hätte.

Diese Tabuisierung der territorialen Fragen bewirkte, dass sowohl das Föderalismusreformgesetz von $2006^{13}$, das der Reformkommission I (wenn auch erst nach Abbruch der Kommissionsberatungen) folgte, als auch das Reformgesetz II im Anschluss an die entsprechende Kommission das bundesstaatliche System sogar eher noch komplizierter und intransparenter machten als es vorher schon war. Die Gründe dafür lagen hauptsächlich darin, dass die erheblichen Ungleichgewichte zwischen den starken und den schwächeren Ländern fortgesetzt wurden und dass die Zahl der Länder unverändert blieb, die mit 16 auf einem so verhältnismäßig engen Territorium wie dem deutschen bei weitem zu hoch war und ist. Der Einwand, dass zum Beispiel die Schweiz auf einem noch wesentlich kleineren Gebiet sogar 26 konstituierende Einheiten (Kantone) hat, ist demgegenüber für sich allein nicht stichhaltig, weil die Ungleichgewichte zwischen ihnen nicht so groß sind wie die zwischen den deutschen Ländern (siehe Tabelle 1).

Die Bemühungen um eine Reform des deutschen bundesstaatlichen Systems sind im Einzelnen insbesondere durch ihre folgenden und eher negativen Ergebnisse gekennzeichnet:

(1) Große Teile von bisher dem Bund zustehenden Gesetzgebungskompetenzen wurden den Ländern übertragen, um die Befugnisse ihrer Parlamente zu stärken. Dies betraf vor allem die Streichung der Rahmengesetzgebung (in Art. 75) und die Schaffung neuer Fassungen der Artikel 72 und 74 über die konkurrierende Gesetzgebungskompetenz des Bundes. Dies wäre in sich noch durchaus kein zu kritisierender Schritt gewesen, aber mit den weiter anhaltenden großen Disparitäten zwischen den Ländern in ihrer Verwaltungs- und Finanzkraft verstärkte dies das Risiko von zu zahlreichen und zu weitreichenden Unterschiedlichkeiten in den Gesetzen von 16 Ländern, die sehr viel leichter tolerierbar wären, wenn deren Zahl kleiner und die verbleibenden Länder dementsprechend größer wären.

(2) Im Bereich der konkurrierenden Gesetzgebungskompetenzen wurde die schwerwiegendste Komplikation durch die Schaffung eines Rechtes der Länder eingeführt, in weiten Bereichen der konkurrierenden Gesetzgebungskompetenzen des Bundes von Bundesgesetzen „abweichende Regelungen“ durch Landesgesetze zu treffen (Art. 72 Abs. 3 GG). Das führt nicht nur zwangsläufig zu rechtlicher Verwirrung, sondern ist außerdem klar zum Vorteil der stärkeren Länder, so dass es die Ungleichgewichte noch weiter verstärkt.

(3) Dasselbe trifft für das neue Recht der Länder zu, auch „abweichende Regelungen“ im Bereich der Verwaltungsorganisation und der Verwaltungsverfahren bei der Durchführung von Bundesgesetzen zu treffen, und in diesem Gebiet (in Art. 84 GG) sind die Regelungen heute sogar noch komplizierter und ebenfalls sogar noch vorteilhafter für die starken Länder, so dass auf diese Weise neue Ungleichgewichte geschaffen und nicht nur bestehende verstärkt werden.

12 Der ehemalige Bundesjustizminister Edzard Schmidt-Jortzig hatte in der Kommission vergeblich solche Versuche unternommen; vgl Uwe Leonardy, Föderalismusreform, a.a.O. (Fn. 9), S. $78 \mathrm{f}$.

13 52. Gesetz zur Änderung des GG vom 28. August 2006, BGBl. I, S. 2034. 
(4) Weiter wurde das gesamte Feld des Finanzausgleichs voll in beiden Reformkommissionen tabuisiert, weil die schwächeren Länder auf der Fortsetzung der Zahlungen an sie durch die stärkeren bestanden, obwohl die weitreichend diskutierten Regelungen inzwischen schon in hohem Maße einebnende Wirkungen hervorgebracht hatten.

(5) Stattdessen wurde den Variationen des Finanzausgleichs (den horizontalen zwischen den Ländern und den vertikalen vom Bund gegenüber den Ländern) ein drittes System zu Gunsten der fünf schwächsten Länder hinzugefügt, durch das sie gemeinsam bis 2020800 Millionen Euro pro Jahr vom Bund und den anderen Ländern erhalten, um ihnen den Weg zur Einhaltung der Schuldenbremse für alle Länder nach diesem Zeitpunkt zu ebnen (Konsolidierungshilfen). ${ }^{14}$

(6) Die Probleme der zahlreichen dicht bevölkerten urbanen Regionen, die durch Ländergrenzen in Stücke geschnitten werden (Art. 29 Abs. 4 und 5 GG), wurden infolge der Weigerung, irgendwelche Territorialreformen überhaupt zu diskutieren, nicht einmal berührt. Mit den Umlandbereichen aller drei Stadtstaaten sowie den Regionen Frankfurt/ Mainz/Wiesbaden, Mannheim/Ludwigshafen und Halle/Leipzig betrifft das erhebliche Teile des Bundesgebiets und ihrer Bevölkerung.

Dementsprechend führte die Tabuisierung der Länderneugliederung alles in allem zu einem „Krückensystem“, das durch die Notwendigkeiten konstruiert wurde, einen Ausgleich für eine nicht angemessene Gleichgewichtsverteilung zwischen den konstituierenden Teilen der Föderation zu schaffen. Anstatt eine Reform des bundesstaatlichen Systems hervorzubringen, haben solche zweifelhaften quasi-Lösungen gemeinsam die Wirkung, alle Länder zu schwächen und zu gleicher Zeit die Macht des divide et impera auf der Seite des Bundes zu stärken.

Aus diesen Gründen hat Deutschland nach wie vor ein territorial stark ungleichgewichtiges föderales System, und der Versuch, diese Ungleichgewichtigkeit als das Zentralproblem aus Reformbemühungen herauszuschneiden, führte zu dem, was an anderer Stelle Reformieren auf der Grundlage des „Shylock-Prinzips“ genannt worden ist ${ }^{15}$ : Ebenso wie der Geldverleiher Shylock in Shakespeares „Kaufmann von Venedig“ begeht man einen fatalen Fehler, wenn man sich in der Lage glaubt, das Herz aus einem lebenden Körper heraustrennen zu können, ohne dabei einen Tropfen Blut zu verlieren. Viele solcher Tropfen, wenn nicht Ströme, sind durch jene Operation der Tabuisierung einer Länderneugliederung in den offensichtlichen Defekten und Kompromisskomplikationen der Reformgesetze von 2006 und 2009 verloren worden. Sie werden auch weiterhin in der Zukunft verloren sein, wenn das Problem einer solchen Reform nicht mit dem Ergebnis eines zumindest angenäherten Arbeitsgleichgewichts zwischen einer kleineren Zahl von besser ausbalancierten Ländern gelöst wird.

Im Hinblick auf all diese politischen und Verfahrensprobleme erscheint es in der Tat als paradox, dass Deutschland der einzige Bundesstaat ist, in dem die Regeln über eine Territorialreform ein klares Bekenntnis zu den Zielen und zu den funktional definierten Zwecken einer solchen Reform enthalten. Art. 29 Abs. 1 GG führt dazu aus: „Das Bundesgebiet kann neu gegliedert werden, um zu gewährleisten, dass die Länder nach Größe und Leistungsfähigkeit die ihnen obliegenden Aufgaben wirksam erfüllen können."

Das ist bis heute „toter Buchstabe“ in der Verfassung geblieben, aber es könnte durchaus wichtige politische Substanz gewinnen, wenn die von der Föderalismuskommission II ent-

14 Art. 143 d Abs. 2 und 3 GG.

15 Vgl. Uwe Leonardy, Föderalismusreform, a.a.O. (Fn. 9), S. 94 f. 
wickelten und daraufhin ins Grundgesetz übernommenen Regelungen über die Schuldengrenze unmittelbar relevant werden und greifen: Diese neuen Regelungen sehen unter anderem in dem neuen Art. 91 d GG vor, dass ein Verfahren des Benchmarking eingeführt wird, um Vergleiche zwischen der Effizienz der Verwaltungskapazitäten der Länder zu ermöglichen und diese zu veröffentlichen. Obwohl dies nur auf einer freiwilligen Basis durchgesetzt werden konnte, könnte dies eine starke Wirkung in der öffentlichen Debatte über die Länderneugliederung entfalten.

\section{Ein Verfahrensmodell zur Ausbalancierung der Rollen des Ganzen und der Teile}

Aber durch welche Art eines Verfahrens könnte nach all diesen Fehlschlägen eine Territorialreform mit angemessen ausbalancierten Rollen des Ganzen und der Teile im Verhältnis zueinander erreicht werden? Wie die vorstehenden Verfassungsanalysen gezeigt haben, ist dies offensichtlich nicht nur ein Problem für Deutschland allein, sondern die Frage würde und könnte sich auch andernorts erheben. Das scheint mithin der Punkt zu sein, an dem die deutsche Fallstudie verlassen werden sollte, obwohl die folgenden Überlegungen sich an die deutsche Erfahrung anlehnen und sie besonders im Blick haben.

Es muss wohl als eine kaum bestreitbare Tatsache angenommen werden, dass die Rolle der gesamten föderalen Wählerschaft als eines abschließenden Schiedsrichters über miteinander im Konflikt stehende regionalen Referenden, wie sie unter den deutschen Regeln bis 1976 existierte, hier aus politischen Gründen kaum wieder herstellbar sein wird ${ }^{16}$, obwohl sie die wirksamste Lösung wäre; ebenso wird sie kaum irgendwo anders eingeführt werden können. Dennoch wird es eine wichtige Forderung sein, dass Einsprüche der von Territorialreformen betroffenen Teile einer Föderation zumindest abgemildert und im Optimalfall auch vermieden werden sollten durch einen Verfassungsmechanismus, der ausreichend Raum für die demokratischen Rechte der Bevölkerung in den betroffenen Teilen gibt, während er aber auch gleichzeitig der Gesamtföderation eine angemessene Rolle zuweist.

Dies würde die Wiederaufnahme von Überlegungen rechtfertigen, die während der Verhandlungen über die Revision des deutschen Verfahrens für eine Territorialreform in den Jahren 1975/76 in ein Konzept gegossen wurden. ${ }^{17}$ Dieses Konzept bestand aus drei Hauptelementen:

(1) Zuerst sollte am Beginn des Verfahrens - nicht mehr an seinem Ende - eine allgemeine Volksabstimmung in der Gesamtföderation über die grundsätzliche, aber zugleich konkretisierte Frage stattfinden, ob eine Territorialreform innerhalb des Gebiets des Bundes angestrebt werden soll. Dieses Referendum sollte auf der Grundlage eines ersten Entwurfs mit Alternativlösungen durchgeführt werden, die aus den Vorschlägen einer unabhängigen Sachverständigenkommission des Bundes hervorgehen. Das Ergebnis dieser Abstimmung hierüber sollte ein für alle Mal die Hauptfrage einer Territorialreform als solcher entscheiden, und es sollte auf diese Weise weitere Dispute über diese Grundfrage vermeiden und befrieden.

16 Wie Arthur Benz es noch 1993 erhofft hatte; vgl. ders., Redrawing the Map? The Question of Territorial Reform in the Federal Republic of Germany, in: Charlie Jeffery / Roland Sturm (Hrsg.), Federalism, Unification and European Integration, London / Portland 1993, S. 38 - 57, S. 51.

17 Initiiert auf der Grundlage eines Entwurfs des Verfassers; vgl. Uwe Leonardy, Neugliederung, a.a.O. (Fn. 9), S. 25 f. 
(2) Zweitens sollten unter der Bedingung, dass dieses Referendum Unterstützung für eine Territorialreform ergeben sollte, alternative und konkrete Modelle durch das direkt gewählte föderale Gesetzgebungsorgan entwickelt werden, und es sollten - wie in Deutschland von Anfang an - diese Modelle nicht der Zustimmung der Zweiten Kammer (in Deutschland also des Bundesrats) bedürfen. Die Modelle sollten auf der Grundlage der Vorschläge der unabhängigen Sachverständigenkommission erarbeitet werden, und sie sollten die regionalen Ergebnisse der allgemeinen Abstimmung zu Beginn des Verfahrens mit berücksichtigen.

(3) Als ein dritter Schritt sollten regionale Referenden in den durch das Reformprojekt unmittelbar betroffenen Gebieten endgültig entscheiden, welche Lösungen innerhalb jener Alternativen bevorzugt werden. Im Gegensatz zu den gegenwärtigen deutschen Verfahrensregeln mit ihren Erfordernissen von doppelten Mehrheiten sollten solche regionalen Referenden in dem neuen Modell aber zu dem Prinzip der einfachen Mehrheiten in den umzugliedernden Gebieten zurückkehren, wie das in allen anderen bundesstaatlichen Verfassungen der Fall ist. Solche klärenden und unmittelbar ergebnisbezogenen regionalen Abstimmungen sollten dann das Verfahren beenden.

Ein Verfahrensmodell dieser Art würde eine große Wahrscheinlichkeit bieten, zu einer zufriedenstellenden und befriedenden Lösung nicht durch eine gesetzlich definierte Rolle der gesamten föderalen Wählerschaft als eines abschließenden Schiedsrichters zu gelangen, sondern dies durch die Überzeugungskraft eines Gesamtreferendums über die Reformfrage überhaupt, verbunden mit der von regionalen Referenden über deren konkrete Lösungsgestaltung, zu erreichen. Solch ein Verfahren würde die berechtigten Interessen der Gesamtbevölkerung eines Staates in einem funktionierenden föderalen System angemessen anerkennen und berücksichtigen ${ }^{18}$, während es sie gleichzeitig mit den legitimen regionalen Ansprüchen auf Selbstbestimmung ausbalancieren würde. Regionalem Widerstand gegen territoriale Reformen in bloßer Verteidigung regionaler "politischer Throne“ würde es dementsprechend nicht mehr weiter gestattet sein, Erneuerungen föderaler Systeme allzu leicht zu blockieren.

Wenn wirksame bundesstaatliche Reformen erzielt werden sollen und traditionelle regionale Strukturen dem im Wege stehen, ist die bloße Beibehaltung solcher Strukturen kein selbst-evidenter Wert im Föderalismus, weil diese immer Wechseln in der Geschichte unterworfen und weil sie selbst nicht selten Produkte bloßer historischer Zufälle gewesen sind. Soweit und solange das Prinzip gegenseitigen Respekts des Ganzen und der Teile zutreffend angewandt wird, dürfen und können fragwürdige Traditionswerte nicht in legitimer Weise Territorialreformen ausschließen und eben dadurch Schaden für den gesamten Bundesstaat herbeiführen.

\section{Durchsetzbarkeit eines neuen Verfahrensmodells in Deutschland}

Das Dilemma für die Durchsetzbarkeit jedes neuen Verfahrensmodells - des oben beschriebenen wie jedes anderen auch - liegt in Deutschland natürlich darin, dass ihm wegen der dazu notwendigen Novellierung des Art. 29 GG und damit einer Verfassungsänderung zwei Drittel des Bundestags und vor allem auch des Bundesrats zustimmen müssten. Problematisch würde das mit Sicherheit vor allem im Hinblick auf die Stimmen der schwachen

18 Nachdem das Bundesverfassungsgericht solche Interessen bereits 1961 als legitim und berechtigt anerkannt hatte; BVerfGE 13, S. 54. 
Länder und damit insbesondere derjenigen werden, die im Zuge der Einführung der Schuldenbremse in Art. 143 d Abs. 2 GG als die Empfänger von Konsolidierungshilfen genannt worden sind, mithin Berlin, Bremen, Saarland, Sachsen-Anhalt und Schleswig-Holstein.

Damit ist aber zugleich auch der Lösungsansatz skizziert: Halten diese Länder die Auflagen des zur Durchsetzung der Schuldenbremse durch Art. 109 a GG geschaffenen Stabilitätsrats nicht ein, ist diese in fortlaufender Überwachung ihrer Haushaltswirtschaft festgestellte Tatsache durch Beschlüsse des Stabilitätsrats festzustellen, und diese Beschlüsse sind zu veröffentlichen. Würden danach die Haushalte der betroffenen Länder von 2020 an nur noch durch Einnahmen aus Krediten ausgeglichen werden können, wäre für sie die Verfassungswidrigkeit ihrer Haushalte gemäß Art. 109 Abs. 3 GG festzustellen, wenn nicht eine der in dieser Bestimmung und in Art. 115 GG festgelegten Ausnahmesituationen vorliegt.

Die Wahrscheinlichkeit ist hoch, dass es zu einer solchen Lage kommen wird; denn angesichts der nur geringen Gesetzgebungskompetenzen der Länder im Steuerbereich (Art. 105 GG) würden Abgabenerhöhungen kaum zu ihrer Vermeidung ausreichen. Die einzige Alternative wären Leistungs- und Personalkürzungen im Ausgabenbereich, und die würden angesichts der überwiegenden Landesaufgaben im Bildungs-, Verwaltungs- und Polizeibereich namentlich in diesen Gebieten vorzunehmen sein, wenn sie ins Gewicht fallen sollen. Es dürfte aber kaum anzunehmen sein, dass sich die Wählerschaft in den betroffenen Ländern mit solchen ihre Lebensverhältnisse intensiv betreffenden Kürzungen abfinden würde. Die Konsequenz wäre, dass das Ende der Lebensfähigkeit dieser Länder faktisch wie politisch evident werden würde.

Diese Folge könnte dann nur noch durch eine grundlegende Neugestaltung des Finanzausgleichs abgewendet werden. Dazu haben die Geberländer aber schon jetzt klar signalisiert, dass sie dem nicht zustimmen würden - und dies wohl schon gar nicht, um damit die Wirkungen der Schuldenbremse für schwächere Länder aushebeln zu lassen. Am deutlichsten hat das der baden-württembergische Ministerpräsident Winfried Kretschmann mit seinem drastischen Hinweis zum Ausdruck gebracht, er halte das System des Finanzausgleichs ohnehin in toto für „bescheuert"19.

Angesichts einer solchen Lage könnten sich die betroffenen Länder einem Verfahrensmodell zur Neugliederung nicht mehr verweigern, das ihre eigene Auflösung implizieren würde. Das würde mithin die durch die gegenwärtige Fassung des Art. 29 GG verschlossene Tür zu den Voraussetzungen für eine Territorialreform des bundesstaatlichen Systems in Deutschland öffnen und damit seine substantielle Erneuerung ermöglichen. ${ }^{20}$

Im Hinblick auf die skizzierten und zu erwartenden Zwangslagen beim Greifen der Schuldenbremse in nur noch sieben Jahren wäre es sachlich wie politisch angebracht, für ihre Bewältigung „bereits“ jetzt angemessene Vorkehrungen zu treffen. Der erste Schritt dazu könnte und sollte in der Einsetzung der im Konzept oben bezeichneten Sachverständigenkommission liegen, weil damit auch rechtzeitig öffentliches Problembewusstsein geschaffen würde.

19 Vgl. Jasper von Altenkirchen, Pack schlägt sich, Pack verträgt sich, in: FAZ vom 22. März 2012, S. 10.

20 So auch der ehemalige Präsident des Bundesverfassungsgerichts Prof. Hans-Jürgen Papier in einer Tagung der Akademie für Raumforschung und Landesplanung am 29. September 2011 in Berlin zum Thema „Neugliederung des Bundesgebietes - oder Kooperation der Bundesländer?"; zu seinen Thesen und denen der anderen Referenten (Wilfried Erbguth, Arthur Benz, Reinhard Timmer, Ernst Hüdepohl und Bodo Hasenritter), http://www.arl-net.de/laenderneugliederung (Abruf am 15. Februar 2013). 